\title{
Visualization of acoustic waves in air and subsequent audio recovery with a high-speed schlieren imaging system: experimental and computational development of a schlieren microphone
}

\author{
Joshua S. Harvey ${ }^{\mathrm{a}, \mathrm{b}, *}$, Hannah E. Smithson ${ }^{\mathrm{b}}$, Clive R. Siviour ${ }^{\mathrm{a}}$ \\ ${ }^{a}$ Department of Engineering Science, University of Oxford, Parks Road, Oxford, UK \\ ${ }^{b}$ Department of Experimental Psychology, University of Oxford, Parks Road, Oxford, UK
}

\begin{abstract}
We present a high-speed single-mirror double-pass coincident schlieren system and corresponding algorithms for the visualization of acoustic waves and recovery of their associated audio signals. Schlieren systems are extensively used to visualize strong shockwaves, such as those from supersonic motion or explosions. Recently, they have also been used to visualize lower amplitude non-linear acoustic phenomena, such as the weak shockwaves arising from impact events including hand claps, belt snaps, and towel cracks. Time-invariant sounds produced by loudspeakers have also been imaged, in one case leading to frequency analysis, although these have been limited to high-frequency signals at very high sound pressure levels. The research presented here shifts the focus from sound-field visualization towards audio signal recovery. A comprehensive exploration of several parameters for imaging sound sources, including frequency, wave form, and amplitude, is presented. In addition, we address for the first time the recovery of phase information, which would be essential for speech intelligibility, and the more general case of non-contact sound field reconstruction. Through image and signal processing, it is shown that audio signals can be recovered from high-speed schlieren video whose acoustic waves appear to be below the limit of visibility, and were previously deemed unrecoverable by virtue of their frequency and sound pressure level. This includes sounds at frequencies and loudnesses relevant for human hearing, producing the first 'schlieren microphone'.
\end{abstract}

Keywords: Schlieren imaging, Sound visualization, Image processing, Visual microphone

\section{Introduction}

In this paper, a novel application of schlieren imaging is presented: a 'schlieren microphone', whereby the optical technique for visualizing refractive inhomogeneities is exploited to indirectly recover audio signals from sound waves. An experimental setup specifically designed for the imaging of acoustic waves relevant to human hearing is combined with software built to recover audio signals from high-speed schlieren footage. This research goes beyond previous attempts to 'visualize' weak shockwaves or acoustic waves: there is an emphasis on signal fidelity in processing, and an evaluation of the limitations of this approach.

Previous attempts to visualize sound with schlieren techniques have mostly been limited to violent or extreme acoustic phenomena such as shock waves, as they give sharp variations of refractive indices in air. These have often relied on significant image processing that would corrupt information contained in the schlieren images. Despite the vast amounts of data present, schlieren images of sound waves have not routinely been analyzed with digital signal processing, with the images instead being inspected and remarked upon qualitatively.

In the current research, the focus is shifted away from visualization and towards signal processing and recovery. Using high-speed photography and an inexpensive schlieren apparatus, it is shown that audio signals can be recovered from schlieren high-speed video, at frequencies and sound pressure levels (SPLs) previously considered too optically

${ }^{*}$ Corresponding author.

Email address: joshua.harvey@pmb.ox.ac.uk (J.S. Harvey) 
weak for schlieren applications. The limitations of this setup are explored through an extensive array of tests, validated by independent microphone measurements, as well as simulated schlieren video.

\section{Background}

\subsection{Theoretical principles of schlieren imaging}

Schlieren imaging is a classical-optics technique for making visible the refractive inhomogeneities in transparent media. Used extensively since the nineteenth century, the technique has found numerous applications in diverse academic and industrial fields such as optics, fluid dynamics [1], aerodynamics, ballistics [2] [3], ultrasonic acoustics [4], epidemiology [5], phonetics [6], and marine biology [7]. The history, optical principles, and much of the modern research surrounding schlieren imaging is explored in Settles' comprehensive book on the topic [8], and Settles and Hargather also also provide a review of recent research [9].

The basic principle of schlieren imaging is that light is precisely focused to a spot by an optical element such as a lens or mirror, whereupon a razor or some other thin device blocks out a portion of the focal spot, after which the remaining light continues into the lens of a camera (see Figure 1). Rays undergoing refraction are deflected while traveling between the source and the focal spot; they consequently are either blocked by the razor instead of forming the image (if deflected in one direction) or avoid the razor instead of being blocked (if deflected in the other direction). The image therefore contains areas of increased or decreased pixel intensity, as a function of the variations in refractive index, $\mathrm{n}$, of the transparent medium being imaged.

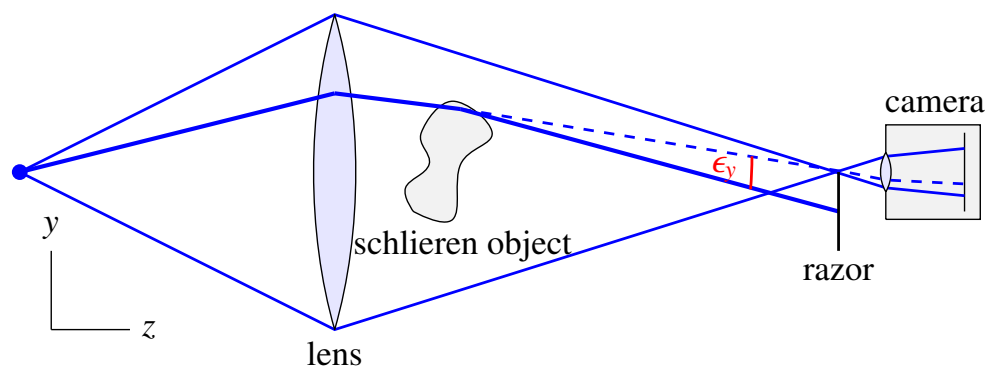

Figure 1: The basic principle of schlieren imaging, shown with a simple one-lens, (horizontally-oriented) knife-edge system. The $y$ axis component of angular ray deflection is indicated by $\epsilon_{y}$.

Using a coordinate system where $z$ is the direction of undisturbed light propagation, and $x y$ are planes orthogonal to this axis, it can be shown that inhomogeneities bend light rays in proportion to their gradients of refractive index, $\mathrm{n}$, in the $x y$ plane. The resulting ray curvatures in the $x$ and $y$ direction are given by:

$$
\frac{\partial^{2} x}{\partial z^{2}}=\frac{1}{\mathrm{n}} \frac{\partial \mathrm{n}}{\partial x}, \quad \frac{\partial^{2} y}{\partial z^{2}}=\frac{1}{\mathrm{n}} \frac{\partial \mathrm{n}}{\partial y}
$$

After integrating once, the $x$ and $y$ plane components of angular ray deflection $\left(\epsilon_{x}\right.$ and $\left.\epsilon_{y}\right)$ are found:

$$
\epsilon_{x}=\frac{1}{\mathrm{n}} \int \frac{\partial \mathrm{n}}{\partial x} \partial z, \quad \epsilon_{y}=\frac{1}{\mathrm{n}} \int \frac{\partial \mathrm{n}}{\partial y} \partial z
$$

Which for a two-dimensional schlieren system of extent $\ell$ along the $z$ axis is:

$$
\epsilon_{x}=\frac{\ell}{\mathrm{n}_{0}} \frac{\partial \mathrm{n}}{\partial x}, \quad \epsilon_{y}=\frac{\ell}{\mathrm{n}_{0}} \frac{\partial \mathrm{n}}{\partial y}
$$

where $\mathrm{n}_{0}$ is the refractive index of the surrounding medium. Therefore, the illuminance level in a schlieren image relates to the first spatial derivative of the refractive index of the transparent medium. A schlieren video consisting of $t$ frames, containing $m$ by $n$ pixels, can therefore be considered as $m \times n$ signals of length $t$, with the intensity of each signal varying proportionally with respect to the relative changes in air pressure along the axis perpendicular to the orientation of the razor. 


\subsection{Previous attempts at schlieren imaging of acoustic waves}

Schlieren imaging is a contrast technique, and although quantitative approaches have been established whereby a system may be calibrated to absolute values of refractive index, the contrast in a schlieren image is an arbitrary function of the particular optical setup and its sensitivity. As such, a certain amount of image post-processing is reasonable, with linear normalization of pixel intensity values to a certain range being the most straightforward and justifiable. Unsharp masking has often been used for visualizations of schlieres (streaks seen in a schlieren image) from shockwaves [10] [11]. While this can produce dramatic images, the processing involved can simultaneously lose information and introduce artifacts into the data recovered from the image. If one is producing schlieren images not just for the purpose of illustration or visualization, but for quantitative analysis of refractive index variation, other processing approaches are required to improve the signal strength of the data.

One potential avenue of processing is the use of digital filters, which can be designed to reduce noise in the image without diminishing the signal, applied to either the temporal or spatial frequencies of intensity variation in the video. Chitanont et al. have implemented this kind of approach for the schlieren visualization of sound fields [12] [13] [14]. The blunt approach of designing a bandpass filter around the expected sound field frequency component can produce attractive images, but is not advisable for two reasons. Firstly, the frequencies present in the sound field must be known beforehand, and secondly, it is difficult to assess to what degree the signal obtained was actually a consequence of the sound field, and how much was simply filtered out of the noise in the image (which will invariably contain some of the sought-after frequencies). A more sophisticated approach uses filter banks. Without relying on exact knowledge of the sound field's frequency content, this approach has been found to successfully improve the signal-to-noise ratio (SNR) in both simulated [13] and real [14] schlieren images of sound fields. The analysis method employed by Chitanont et al. allows for frequency component analysis of the raw and filtered video, but does not permit the phase-alignment of signals required for audio recovery.

\subsection{Other imaging modalities for acoustic wave visualization and audio recovery}

Other imaging modalities have also been used to visualize acoustic waves and sound fields. In the National Physical Laboratory review of acousto-optical sound field visualization [15], schlieren imaging is mostly overlooked in favor of coherent light techniques such as laser doppler anemometry (LDA) [16, 17, 18, 19], laser doppler velocimetry (LDV) [20, 21], and photon correlation spectroscopy (PCS) [22, 23]. LDA and LDV have proved especially promising, with both techniques having been applied to the calibration of pressure microphones. However, these methods require lasers and other high-quality optical elements such as beam-splitters, so there are incentives for developing alternative modalities with less-stringent practical limitations. Existing methods may also require seeding particles to be introduced into the sound-carrying medium. Additionally, methods such as LDV may require repeated measurements of acoustic signals, prohibiting the mapping of transient sound fields.

While previous schlieren visualizations of sound fields may be useful for some acoustic applications, the various approaches tend not to prioritize signal fidelity and recovery. Once this is considered, it becomes clear that a schlieren video effectively comprises many hundreds or thousands of signal detectors, with every pixel theoretically sampling from the first spatial derivative of the sound field perpendicular to the razor's alignment. While each pixel's time series has a vanishingly small SNR, owing to the weak changes in refractive index caused by acoustic waves in the normal range of human hearing, if these numerous, related, time series could be combined, the limitation of each pixel's SNR could be overcome, producing a single one-dimensional time series signal from each video. In theory, integration of this signal would then recover the original audio signal in the acoustic wave, such as would be detected by a conventional microphone. This approach bears similarities to the 'visual microphone' developed to recover sound from high-speed video of everyday household objects [24].

In this paper, some simple processing and filtering is applied (such as to remove frequencies outside the range of human hearing), but a higher SNR is primarily achieved by the combining (averaging) of numerous data series. The resultant 'schlieren microphone' is then comprehensively evaluated through experimental tests, and the findings are validated through simulation work. Two different avenues of data recombination are presented, the first using a geometric model of the sound field and its acoustic waves derived from a point source assumption, and the second using a data-driven autocorrelation approach that more faithfully captures the actual propagation of acoustic waves. 


\section{Experimental setup}

A representation of the single-mirror double-pass coincident schlieren apparatus used to collect all the data featured here is shown in Figure 2. The system has been optimized to be both affordable and highly sensitive, using a double-pass setup first described in 1933 by Taylor and Waldram [25], for which sensitivity is double that of a single-pass schlieren method, as light is deflected by refractive inhomogeneities for both the incoming illuminator and outgoing analyzer beams.

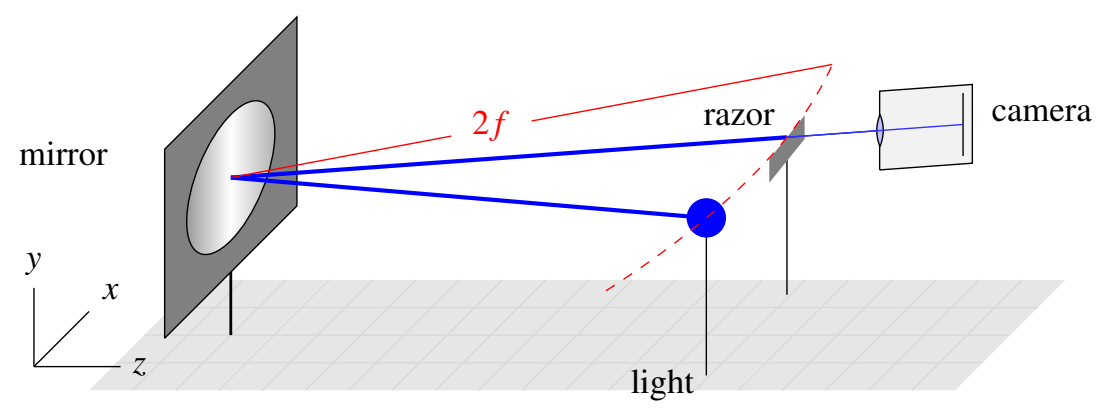

Figure 2: The single-mirror double-coincident setup for schlieren imaging. The angle between the illuminator and analyzer beams is exaggerated for pictorial clarity.

As shown in Figure 2, just four components are required: a spherical mirror, in this case a $150 \mathrm{~mm}$ diameter focusing mirror with a focal length of $1.5 \mathrm{~m}$; a light source; a razor; and a camera for collecting the image. While previous acousto-optic research exploring sound field visualization has mostly required coherent light in the form of lasers [15], the schlieren system presented here uses 'white' non-coherent light emitted from a XENON NOVA® 300. The light source, by use of a liquid light guide, is placed at the mirror's radius of curvature, i.e. twice the focal length distance, $f$, such that $r=2 f$. Diverging light from the source illuminates the mirror, returning off-axis to a focus spot also at the mirror's radius of curvature, whereupon it is partially blocked by a razor. The degree of off-axis divergence between the illuminator and analyzer beams is minimized to under $10 \mathrm{~mm}$ (and exaggerated in the figure) to reduce distortion in the image. The unblocked light continues past the razor into the lens of a camera, where it is focused to produce an image. For the imaging of acoustic waves, high-speed photography is necessary; here a Photron FASTCAM SA5 was used, fitted with a Nikkor $200 \mathrm{~mm}$ lens.

Several parameters were varied during data collection, to explore the potential and limits of acoustic wave visualization and signal recovery; these are summarized in Table 1 . For all conditions, sounds were produced by a Scanspeak D2604/833000 Tweeter. Positioned directly in front of the mirror, the speaker output signals produced by either a function generator or an online application capable of producing various waveforms, and was driven by an audio amplifier system to a range of volumes. Hence, the SPL was varied between conditions to experimentally determine limits of visibility, being read by a Tenma sound-level meter positioned $50 \mathrm{~mm}$ away from the diaphragm of the speaker. For the higher frequency sound waves tested this distance is sufficient for measurements to be in the far-field, where SPL varies according to the inverse square law, and hence characterizes the whole sound field. While this assumption cannot be upheld for lower frequency sound waves $(<10 \mathrm{kHz})$, the measurement still provides an indication of SPL in the region of air being imaged. All SPLs are given in $\mathrm{dB}$ according to the standard convention that $0 \mathrm{~dB}=$ auditory threshold at $1 \mathrm{kHz}$. Waveforms were generated as either sine waves or square waves, for a range of frequencies between $110 \mathrm{~Hz}$ and $25 \mathrm{kHz}$. However, as the acoustic wave outputs are limited by the frequency response of the speaker (which sharply drops off at around $40 \mathrm{kHz}$ ), it should be noted that at higher frequencies fewer harmonics of square waves are feasible. Therefore while a $20 \mathrm{kHz}$ square wave may have been generated and sent to the speaker, one would expect a sound field containing only a $20 \mathrm{kHz}$ sine wave. Additionally, it was not possible to image acoustic waves between 4 and $5 \mathrm{kHz}$, as these appeared to set up corresponding resonant vibrational modes in the mirror itself, distorting the image.

All of the footage collected by varying the parameters of both the speaker output and imaging setup is published open-source, freely available for download [26]. 


\begin{tabular}{||c|c||}
\hline Parameter & Values \\
\hline \hline Waveform & Sine or square wave \\
\hline $\mathrm{SPL} / \mathrm{dB}$ & $\begin{array}{l}80,85,90,95,100, \\
105,110,115,120\end{array}$ \\
& $\begin{array}{l}1.11,0.22,0.44,1,2, \\
5,7,10,15,20,25\end{array}$ \\
\hline Frequency $/ \mathrm{kHz}$ & $40,50,60 \times 1000$ \\
\hline Framerate $/ \mathrm{fps}$ & $4,7,10,16,20$ \\
\hline Exposure time $/ \mu \mathrm{s}$ & \\
\hline
\end{tabular}

Table 1: Imaging and acoustic wave parameters varied for data collection.

\subsection{Defining the limit of visibility}

The 'limit of visibility' is a term that has been employed without much explanation or definition in the discussion of schlieren images. It is broadly interpreted as the minimum variations in contrast of an image required to perceive the differentials in refractive index present. This is a function of both the refractive index variations in the transparent medium and the sensitivity of the schlieren apparatus, as well as imaging parameters pertaining to the camera.

For acoustic waves in the range of human hearing, a highly sensitive schlieren apparatus is required to give clear contrast variations in the individual images collected. However, when reviewing unprocessed video footage it becomes clear that there is a visible, yet subtle, 'shimmering' present for many acoustic waves of weaker SPLs, for which variations are not apparent in static images. This illustrates a perceptual mechanism of the human visual system, which can extract motion information when seemingly random intensity values have a correlation in their movement. This can be accomplished even when there is a strong background illuminant dominating the image, with the visual system adapting to very low-frequency variations in background intensity, analogous to a high-pass filter. Here, quantitative data will be recovered from these sequences.

\section{Image processing and data analysis}

\subsection{Visibility of acoustic waves and image denoising}

Although the motion of acoustic waves propagating through the image is perceptible when viewing the raw schlieren images as a video, for individual frames it is not possible to clearly discern the contrast variations. There are several post-processing routes to improving visibility, each with different advantages and disadvantages.

Unsharp masking is the simplest way to improve the visibility of intensity variations. However, due to the sensitivity of the schlieren system, there are many other sources of intensity variations such as thermal currents and the grinding imperfections of the mirror. The results of unsharp masking with the inbuilt MATLAB function imsharpen can be shown in Figure 5 a.

Spatial filtering with 2D Gaussian kernels also did little to improve the visibility of the acoustic waves in the image. High-pass and low-pass Gaussian filters were designed based on the expected spatial frequency of the sound waves in the image, as shown in Figure 3. Although some improvements can be seen, especially when the footage is viewed as a video, many of the artifacts in the image caused by thermal currents have a similar spatial frequency to the variations in intensity caused by acoustic waves.

In contrast, temporal high-pass filtering (Figure $4 \mathrm{~d}$ ) results in a marked improvement of visibility, as thermal currents move slowly compared to propagating sounds. The resultant image still contains high-frequency noise from the camera, which can be removed by subsequent spatial filtering (Figure 5 e). High-pass temporal filters, either in the form of an 'ideal' filter (zeroing the lowest bins of the signal in the frequency domain) or a Butterworth filter, readily give visible results for many signals.

The SPL of acoustic waves is a major limiting factor for their visibility. Frequency is another factor; waves of higher frequency are more visible as refractive index gradients are greater. Even with the spatial and temporal filtering discussed above, only acoustic waves above certain frequencies and registering certain SPLs can be made visible, as shown in Figure 6. 

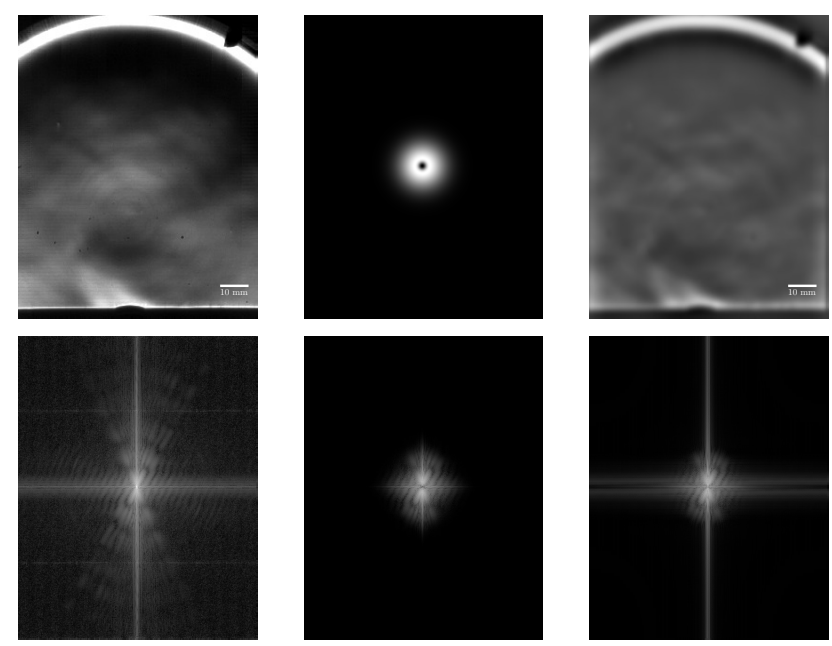

Figure 3: Filtering a frame of schlieren video with 2D Gaussian filters, $20 \mathrm{kHz}$ sine wave, 50,000 fps. Upper panel, left: an unprocessed frame; center: the Gaussian filter response in 2D Fourier space; right: the frame after convolution with the filter. Lower panel, left: FFT of the raw image; center: the product of the raw image FFT and the filter in Fourier space, right: FFT of the resultant image.

\subsection{Data recombination through modeling of the sound field}

The signal of each pixel in the sound field image can be defined by a shift in time (or phase, for periodic signals) and change in amplitude relative to the source signal. While some limited frequency component analysis has previously been performed on high-speed schlieren video of acoustic waves [14], this did not address the phase realignment of signals from individual pixels required to recover an audio signal. Magnitude spectra (e.g. spectrograms) are often presented without phase data for discussions of sound, and particularly phonetics and the perception of speech. However, it has been found that phase can contribute significantly to speech intelligibility [27], especially for weak or noisy signals [28].

If the sound field is modeled as spherically emanating from a point source, the time shift for a pixel's time series corresponds to its radial distance from the sound source. The amplitude can then be modeled by the spherical decay of the acoustic wave in three-dimensional space. For each pixel, $I_{x_{i}, y_{i}}$, at a radial distance $r_{i}$ from a sound source modeled at $x_{0}, y_{0}$, the time shift $\tau_{i}$ of the signal and its relative amplitude $A_{i}$ are given by:

$$
\begin{aligned}
r_{i} & =\sqrt{\left(x_{i}-x_{0}\right)^{2}+\left(y_{i}-y_{0}\right)^{2}} \\
\tau_{i} & =\frac{r_{i}}{c \cdot \psi}, \quad A_{i}=\frac{1}{r_{i}^{2}}
\end{aligned}
$$

where $\psi$ is the scale of the schlieren video in pixels per meter and $c$ is the wave speed in meters per second.

This point-source assumption can therefore be used to produce a phase map for each schlieren image, allowing the time series from pixels to be combined once shifted back in phase. An example phase map is given in Figure 10. The data can then be shifted in a pixelwise manner so that their time series are all aligned. Taking the mean for each realigned frame will then give a single time series signal, with a sampling frequency equal to the frame rate of the footage.

However, compared to the spatial resolution of the image, the temporal resolution of the schlieren video with respect to acoustic waves is poor. This is illustrated in Figure 7. To retain the high resolution of the data within each frame, the data can be reorganized by radial distance rather than pixel index, ordered by increasing distance from the sound source, and resampled to give a uniform distribution of data. The intensity values in images across frames can then be combined according to the time shift between frames (i.e. the frame rate, $\lambda$ ). The time shift for any intensity value $\tau$ is therefore a function of both its radial position $r_{i}$ and its frame $k_{n}$ : 

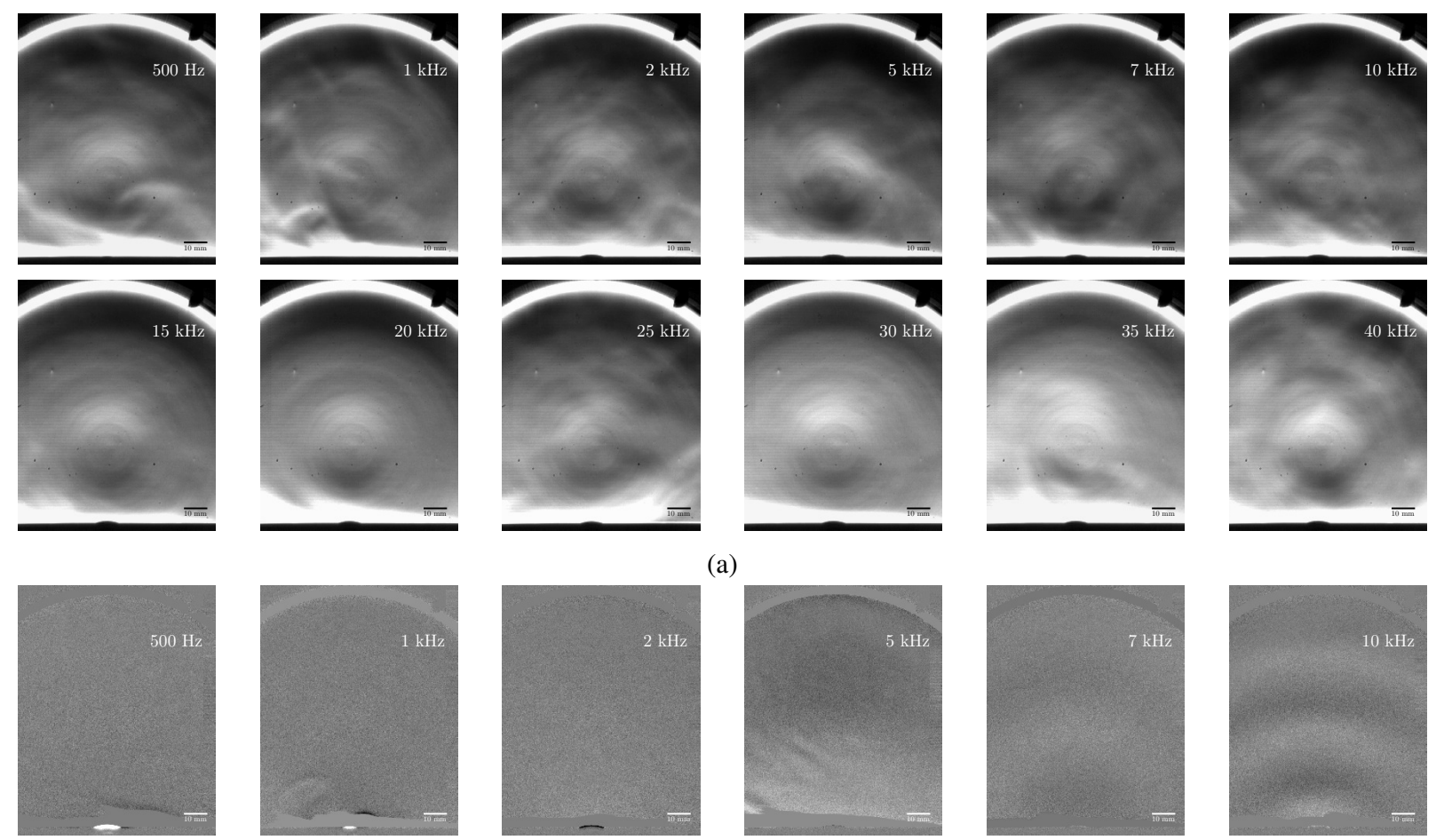

(a)
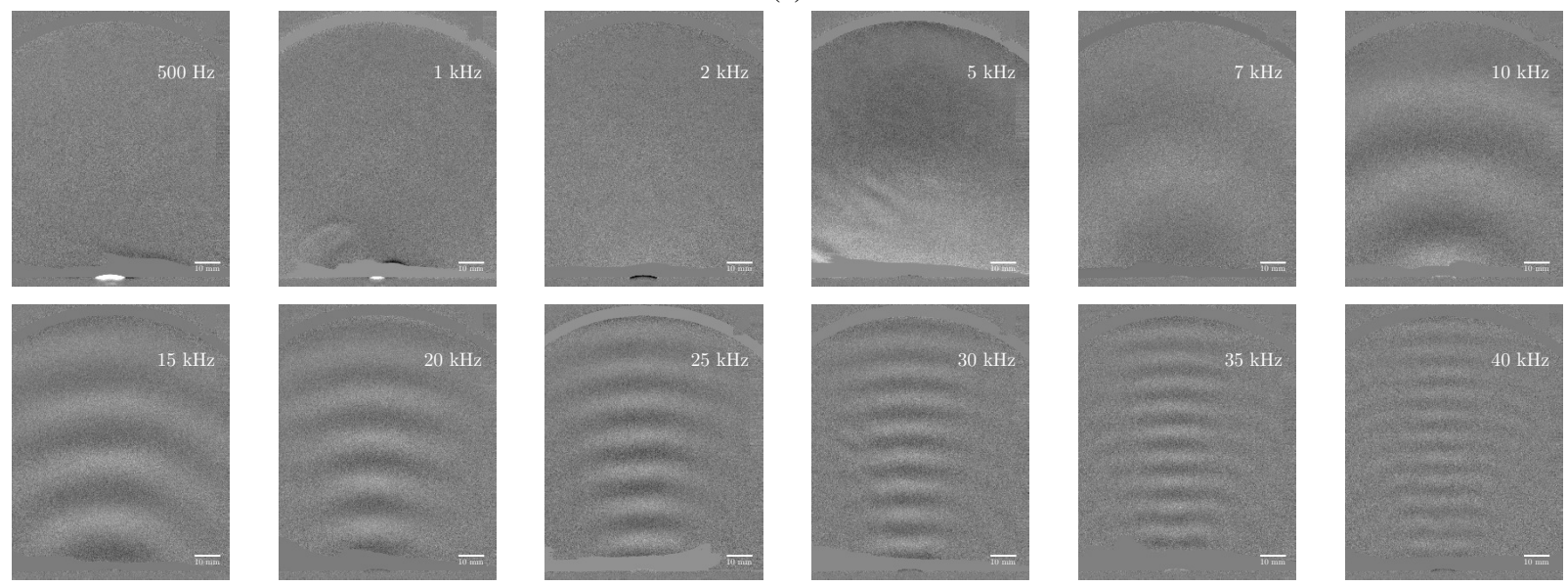

(b)

Figure 4: (a) Raw footage and (b) footage after a high-pass temporal filter (cut off $=100 \mathrm{~Hz}$ ) has been applied. Top left to bottom right: $500 \mathrm{~Hz}$ $(115 \mathrm{~dB}), 1 \mathrm{kHz}(120 \mathrm{~dB}), 2 \mathrm{kHz}(120 \mathrm{~dB}), 5 \mathrm{kHz}(120 \mathrm{~dB}), 7 \mathrm{kHz}(120 \mathrm{~dB}), 10 \mathrm{kHz}(120 \mathrm{~dB}), 15 \mathrm{kHz}(120 \mathrm{~dB}), 20 \mathrm{kHz}(115 \mathrm{~dB}), 25 \mathrm{kHz}(100$ $\mathrm{dB}), 30 \mathrm{kHz}(105 \mathrm{~dB}), 35 \mathrm{kHz}(90 \mathrm{~dB}), 40 \mathrm{kHz}(75 \mathrm{~dB})$.

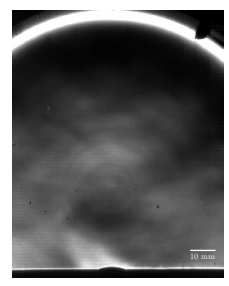

(a)

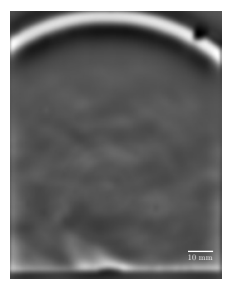

(b)

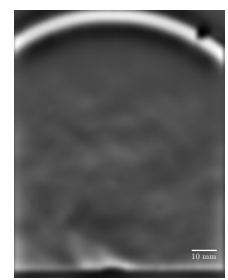

(c)

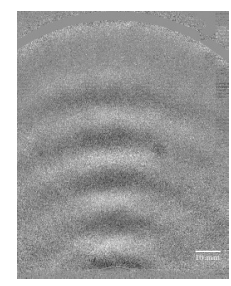

(d)

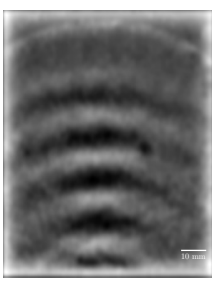

(e)

Figure 5: Different post-processing for a $20 \mathrm{kHz}$ sine wave, $115 \mathrm{~dB}$ : (a) raw footage; (b) after unsharp masking; (c) after spatial filtering with a 2D Gaussian kernel; (d) after a high-pass temporal filter is applied; and (e) with temporal and spatial filtering. Note that the best visibility is obtained using the latter post-processing method (e).

$$
\tau\left(r_{i}, k_{n}\right)=c \cdot r_{i}+\lambda \cdot k_{n}
$$

With every data point from the footage now identified by a time shift in signal from the sound source, a one 

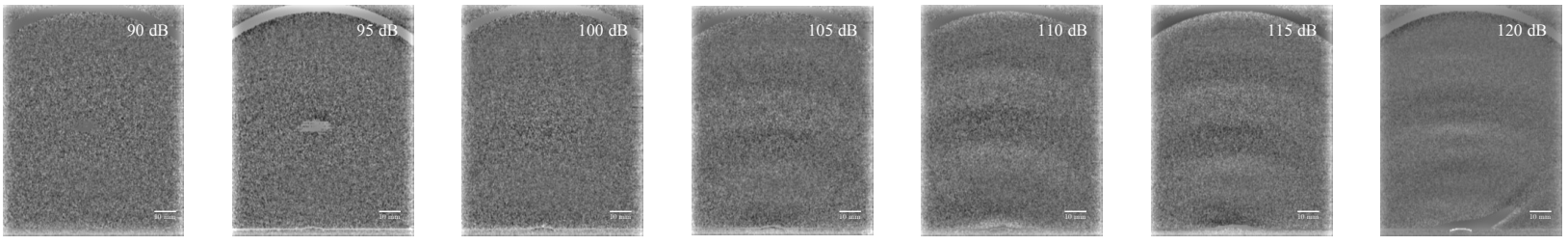

Figure 6: Visibility of acoustic waves is dependent on a high enough SPL for a given schlieren sensitivity. Left to right: a $10 \mathrm{kHz}$ square wave measured at SPLs of 90, 95, 100, 105, 110, 115 and $120 \mathrm{~dB}$.

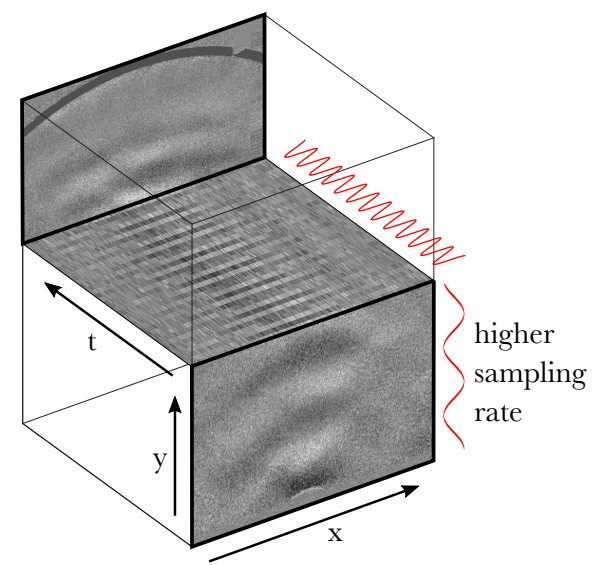

Figure 7: A visualization of footage taken of a $20 \mathrm{kHz}$ sine wave. The first and last frames of a 35 frame sequence are shown, connected by a single row of pixels varying through time. Approximate variations in pixel intensity through the center of the image are shown in red. It can be seen that the video has a far higher sampling rate spatially than temporally.

dimensional time series is obtained with a very high sampling rate, as illustrated in Figure 8. Smoothing, by cubic spline interpolation or a moving average, can then be used to obtain a signal at a desired sampling rate with a high SNR.

\subsection{Data recombination through autocorrelation of signals}

While modeling the sound field as circular waves propagating radially from a point source gives good results, it is clear that it does not perfectly account for the actual propagation of acoustic waves. As can be seen in Figure $4 \mathrm{~b}$, the point source approximation is increasingly inaccurate as frequency of the acoustic waves increase, because waves spread out less and retain more of the flat geometry of the extended sound source (i.e. the speaker).

For schlieren videos with sufficiently strong contrast changes throughout the sound field, a truer mapping of wave propagation can be obtained through the cross-correlation of pixel time series. First, the time series data for each pixel, $I_{x, y}$, are upsampled by an integer rate $L$ with an interpolation filter:

$$
U_{x, y}[j+n L]=\sum x[n-k] \cdot h[j+k L]
$$

where $h$ corresponds to the impulse response of the low-pass filter.

This upsampling will allow each time series to be phase shifted at a higher temporal resolution than that of the frame rate. Then, the data for every pixel in the image are cross-correlated to a dataset, $M_{\mu, y}$, obtained by averaging a small number of high-contrast pixels whose upsampled data are already aligned (i.e. neighboring pixels taken from a single row in the center of the wavefront). For each pixel the signal lag, $\gamma$, giving rise to the maximal cross-correlation is then converted as a function of upsampling rate and frame rate to give the phase shift (as the signal is periodic): 

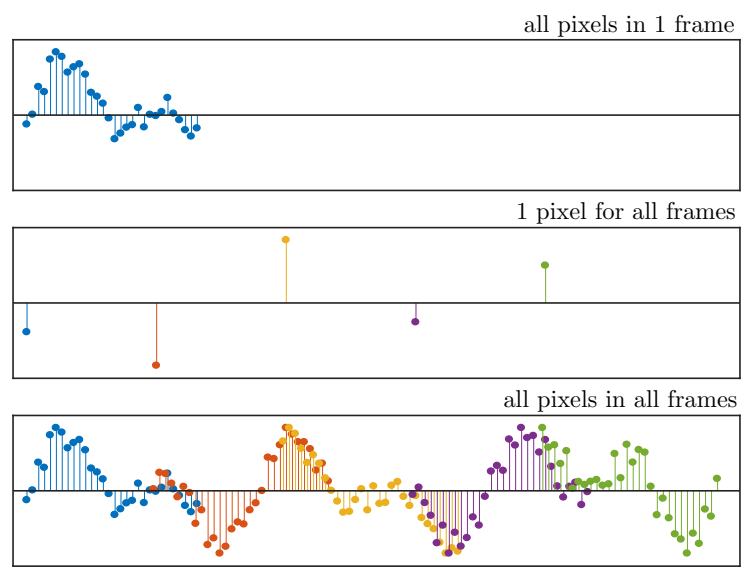

Figure 8: An illustration of the effective sampling rates for intra-frame spatial resolution and inter-frame temporal resolution. The intensity values for pixels in a single frame give dense data within a limited time period. Depending on the frame rate, these can overlap with data across other frames, and be combined by calculating the time shift between frames.

$$
\begin{aligned}
M_{\mu, y} & =\sum_{\mu=-n}^{n} \frac{1}{2 n+1} U_{\mu, y} \\
\phi_{x, y} & =\underset{\gamma}{\arg \max }\left(\left(U_{x, y} \star M_{\mu, y}\right)(\gamma)\right) \frac{1}{L \cdot \lambda}
\end{aligned}
$$

where $\phi_{x, y}$ is the phase shift for pixel $I_{x, y}$ in the footage. An example phase-shift map is shown in Figure 10 of the Results section.

This phase-shift map is converted to a map of each pixel's time shift by adding the maximal lag multiplied by the wave crest index, $w$, of the pixel, ultimately producing a more accurate time shift map of the video:

$$
\tau_{x, y}=\phi_{x, y}+\max (\gamma) \cdot w_{x, y}
$$

\section{Simulated schlieren video of acoustic waves}

Generating simulated schlieren video from specified signals and noise can validate the analysis of experimentderived footage. A sound field can be modelled as sinusoidal waves propagating from a point source. The amplitude of pressure, $P$, throughout the sound field at time $t$ and a radial distance $r$ from the source is given by:

$$
\begin{aligned}
& \qquad \begin{aligned}
P(r, t) & =\frac{P_{0}}{r^{2}} \exp \{i(\kappa r-\omega t)\} \cdot \exp \{\phi(\omega)\} \\
& \propto \frac{1}{r^{2}} \exp \{i(\kappa r-\omega t)\} \\
\text { where } \omega & =2 \pi f
\end{aligned}
\end{aligned}
$$

For a harmonic series of sine waves, with the $h$-th harmonic corresponding to an angular frequency $\omega_{h}$, the pressure function for the total series approximated to $\sigma$ harmonic components is given simply by the sum of each harmonic:

$$
P(r, t)=\sum_{h=1}^{\sigma} P\left(\omega_{h}, r, t\right)
$$


For a schlieren image, $S$, the first derivative of pressure in one direction is required, obtained by differentiating with respect to $x$. For a given waveform:

$$
\begin{aligned}
S & =\frac{\partial P}{\partial x}=\frac{\partial P}{\partial r} \cdot \frac{\partial r}{\partial x} \\
\frac{\partial P\left(\omega_{h}, r, t\right)}{\partial r} & =H_{h}\left(\frac{-2}{r^{3}}+\frac{i \kappa}{r^{2}}\right) \exp \{i(\kappa r-\omega t)\} \\
\frac{\partial P\left(\omega_{h}, r, t\right)}{\partial r} & =H_{h}\left(\frac{\kappa \cos \left(\kappa r-t \omega_{h}\right)}{r^{2}}-\frac{2 \sin \left(\kappa r-t \omega_{h}\right)}{r^{3}}\right) \\
\frac{\partial r}{\partial x} & =\frac{x}{\sqrt{x^{2}+y^{2}}}
\end{aligned}
$$

where $H_{h}$ is the Fourier coefficient for the $h$-th harmonic component. Terms can be expressed either as complex numbers (eq. 14) or the sums of sines and cosines (eq. 15, showing only real components). Combining eq. 15 and eq. 16 , the component from each harmonic in a sound field is therefore:

$$
S\left(\omega_{h}\right)=H_{h}\left(\frac{\kappa \cos \left(\kappa r-t \omega_{h}\right)}{r^{2}}-\frac{2 \sin \left(\kappa r-t \omega_{h}\right)}{r^{3}}\right) \cdot\left(\frac{x}{\sqrt{x^{2}+y^{2}}}\right)
$$

With the total schliere contrast for a wave approximated to $\sigma$ harmonic components given by:

$$
S(r, t)=\sum_{h=1}^{\sigma} S\left(\omega_{h}, r, t\right)
$$

Once calculated, simulated schlieren images may then be combined with zero-mean Gaussian noise to simulate the heat noise of the camera's image sensor. Results from the simulated schlieren analysis confirmed that the software was performing as desired, and example images can be found in Figure 9.

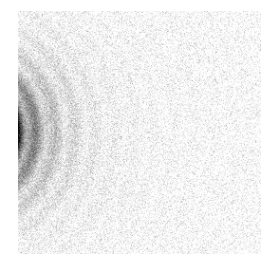

(a)

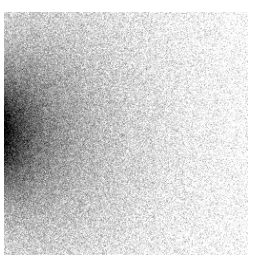

(b)

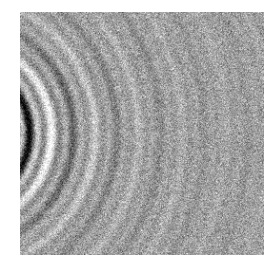

(c)

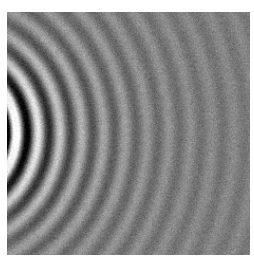

(d)

Figure 9: Simulated schlieren images, produced with different waveforms and signal-to-noise ratios. (a) $440 \mathrm{~Hz}$ square wave, 25 Fourier components, $\mathrm{SNR}=0$. (b) $1 \mathrm{kHz}$ sine wave, $\mathrm{SNR}=4$. (c) $10 \mathrm{kHz}$ 'square' wave (only 2 Fourier components), $\mathrm{SNR}=-2$. (d) $20 \mathrm{kHz}$ sine wave, $\mathrm{SNR}=10$

\section{Results}

Figure 10 shows the overall work flow of the schlieren microphone; from high-speed schlieren footage of a sound field to the recovered audio signal. Both the point-source assumption model and the auto-correlation of signals are shown as possible routes to relate and combine each pixel's time series data into a single signal.

Three example results are provided in Figure 11, showing the recovered audio for both high-frequency and lowfrequency waveforms, and both sine and square waveforms. For the data presented here, signal processing and analysis was performed on a Macbook Pro (Early 2015) with 16 GB RAM, running MATLAB 2017a. For a single schlieren video file of 1000 frames, the total time required from data import to audio export was approximately ten minutes. 


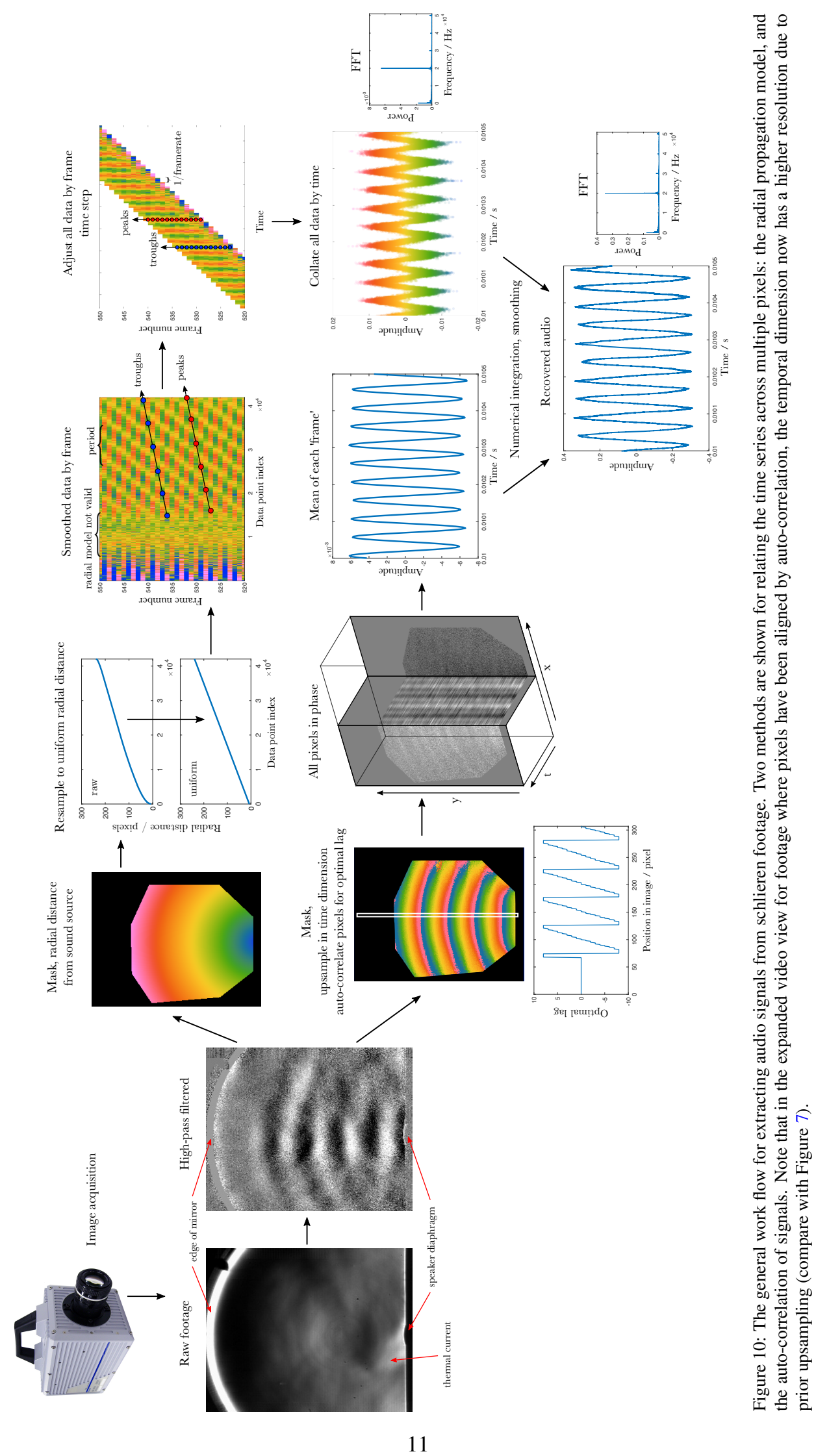



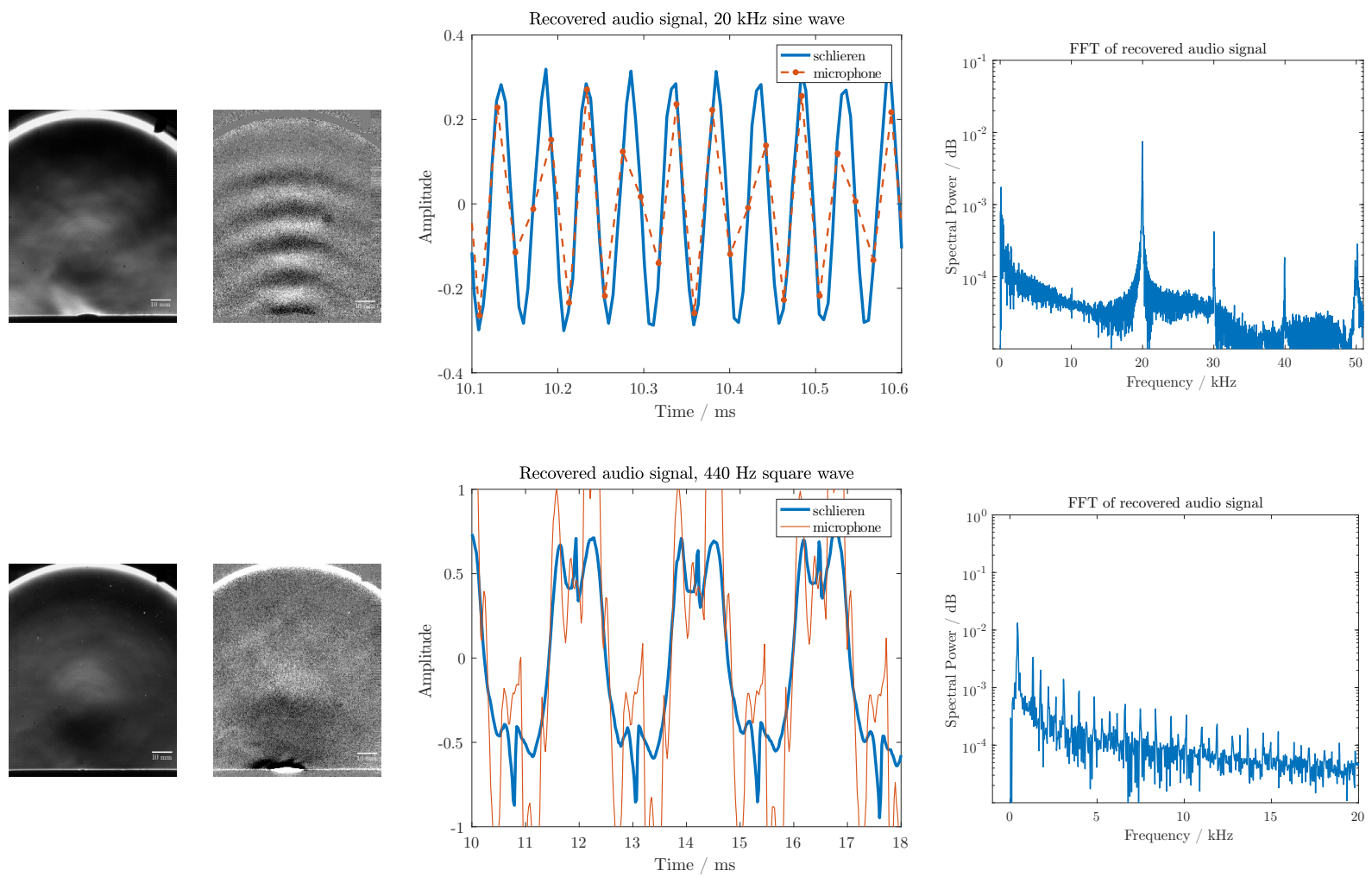

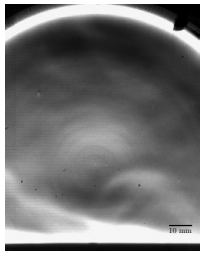

(a)

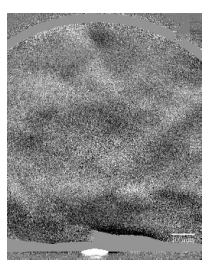

(b)

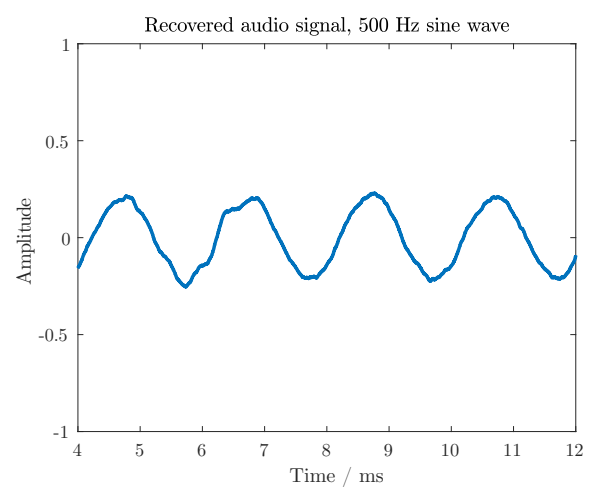

(c)

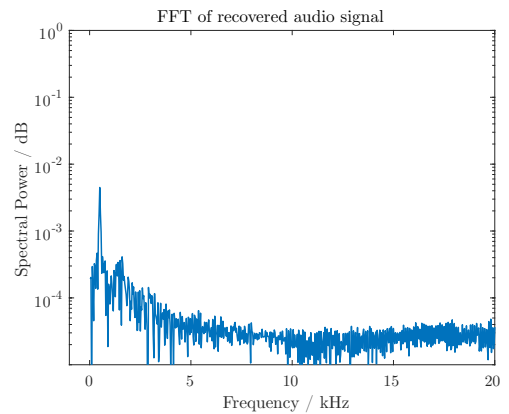

(d)

Figure 11: Examples of different frequencies and waveforms; (a) a frame from the raw schlieren footage; (b) the same frame after filtering; (c) the audio signal recovered by the schlieren microphone (blue) and a conventional microphone (red); (d) the spectral makeup of the recovered audio. Top panel: $20 \mathrm{kHz}$ wave recorded at $115 \mathrm{~dB}$. It is clear that the schlieren microphone has a far higher sampling rate than a conventional microphone, which is visibly undersampled. Middle panel: $440 \mathrm{~Hz}$ square wave at $120 \mathrm{~dB}$. Many Fourier components, corresponding to the odd harmonics of the square wave, are recovered. Lower panel: $500 \mathrm{~Hz}$ sine wave at $115 \mathrm{~dB}$. Audio signals were recovered by use of the point-source assumption model.

\subsection{Visibility of acoustic waves is dependent on frequency, waveform, and amplitude}

In accordance with previous literature and mathematical models, the limit of visibility was found to be dependent on both the amplitude and frequency of the acoustic wave, with greater amplitudes and higher frequencies giving rise to increasingly clear contrast differentials.

As discussed above, there is no strict definition of visibility, and while contrast differentials may be visible when footage is inspected as a video, individual schlieren photographs may not appear to feature any signal. Figure 12 
shows the approximate limits of visibility as a function of two parameters-amplitude and frequency.

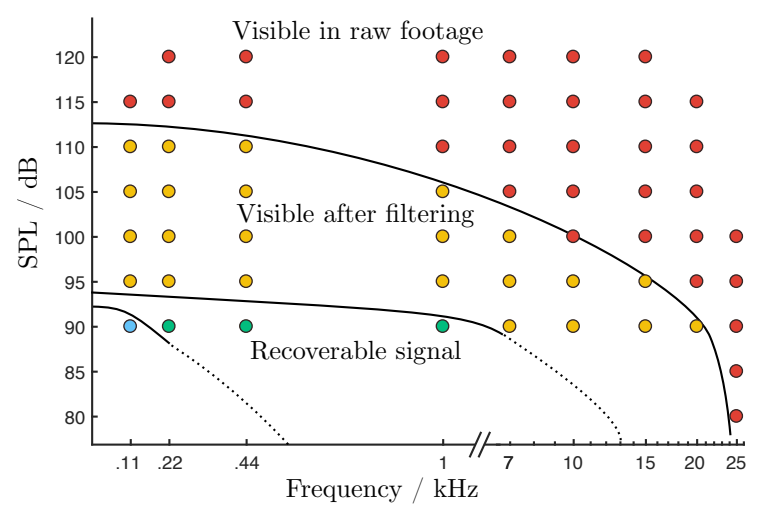

Figure 12: Summary of results, showing which samples are visible in raw and processed footage, or only recoverable after spatio-temporal processing. Where example footage is available, markers have been color-coded according to data quality (visible in raw footage $=$ red; visible after filtering = yellow; recoverable signal after processing $=$ green; no recoverable signal $=$ blue). These findings are particular to the schlieren system used for imaging, and the parameter space of visible and recoverable waveforms may be extended with a schlieren system of greater sensitivity or reduced noise.

However, more objective evaluations of the recoverability of the audio signal can be found in metrics such as route mean square (RMS) and SNR. Figure 13 illustrates how the RMS for each pixel throughout the video can be used to visualize where the sound field is most visible. Notably, lower frequency acoustic waves, down to $110 \mathrm{~Hz}$, may still yield audio signals of high SNR even at lower SPLs, beyond the limits of visibility, as shown in Figure 14. By comparing with Figure 12, it is clear that the visibility of acoustic waves in raw and/or filtered footage is not a good predictor of the recoverability of an audio signal and its SNR across different frequencies. This makes intuitive sense when the limits of the human visual system are taken into consideration. A schlieren video of a low-frequency wave may have just as strong contrast differentials as a high-frequency wave, but as these variations in contrast are distributed across many frames, they may go unnoticed. This is due to the contrast sensitivity function (CSF) of the human visual system, which has a bandpass filter for spatial frequency: visibility of image features drops both above and below peak sensitivity at 3 to 4 cycles per degree of visual angle. This is illustrated by a Campbell-Robson contrast sensitivity chart [29], shown in Figure 15, using the CSF described by Mannos and Sakrison [30]:

$$
A\left(f_{r}\right) \approx 2.6\left[0.0192+0.114 f_{r}\right] \exp \left\{-\left(0.114 f_{r}\right)^{1.11}\right\}
$$

where $A\left(f_{r}\right)$ is the contrast sensitivity function for an image feature of spatial frequency $f_{r}$, defined in cycles per degree of visual field.

RMS maps for $20 \mathrm{kHz}$ wave, varying SPL

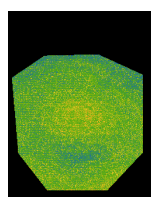

$90 \mathrm{~dB}$

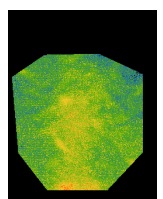

$105 \mathrm{~dB}$

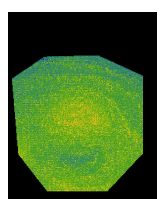

$95 \mathrm{~dB}$

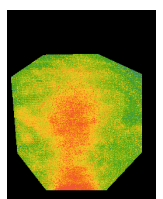

$110 \mathrm{~dB}$

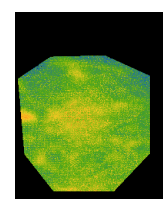

$100 \mathrm{~dB}$

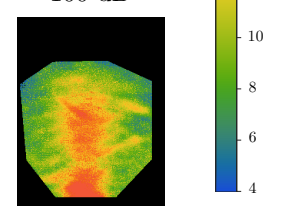

$115 \mathrm{~dB}$

$\operatorname{RMS} \times 10^{-3}$

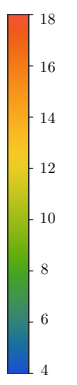

Figure 13: Variations of pixel RMS values according to acoustic wave amplitude. 


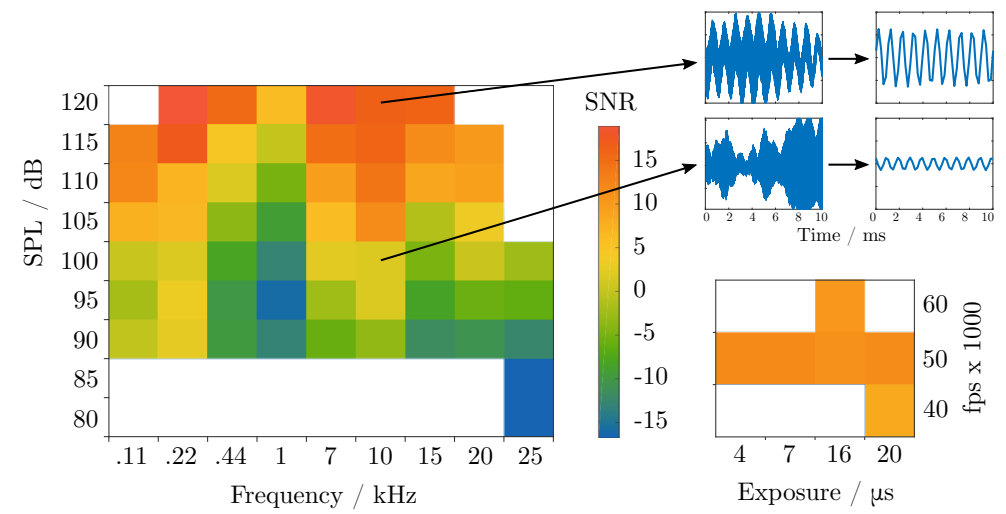

Figure 14: Plots of SNR to evaluate the quality of recovered audio signal. The audio signals recovered from footage of a $10 \mathrm{kHz}$ waveform, measured at two SPLs are shown to illustrate the different SNRs. Data are also shown for the effect of varying imaging parameters-both exposure time and frame rate-although this had a minimal effect on the SNR of audio recovered from a $10 \mathrm{kHz}$ square wave measured at a SPL of $105 \mathrm{~dB}$.

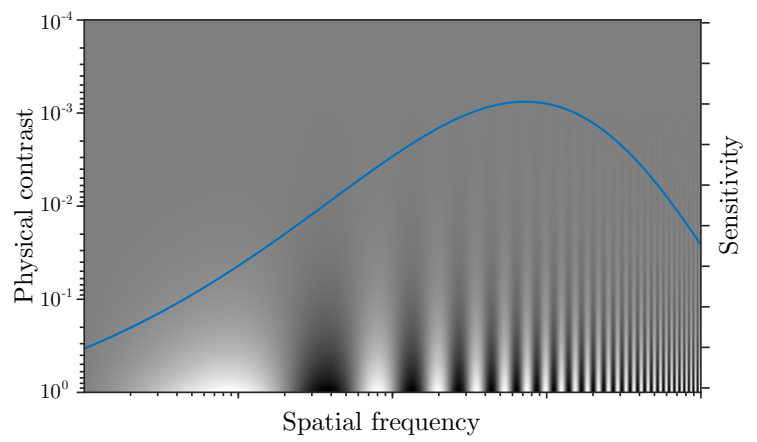

Figure 15: The human visual system is limited by a contrast sensitivity function, illustrated here. For different spatial frequencies of image contrast variation, illustrated here by a sine wave of logarithmically-increasing frequency, the sensitivity of the human visual system (indicated by the right axis, and shown in blue) varies for equivalent changes in physical intensity contrasts (indicated by the left axis). The overall effect is an envelope of visibility of the sine wave, with a peak sensitivity at between 3 and 4 cycles per degree of visual field. Sensitivity according to the CSF is overlaid in blue. (This plot will be subject to image size and viewing distance.)

\subsection{Impact of imaging parameters on recovered data}

With high-speed imaging, there is a trade-off between temporal and spatial resolution, as higher frame rates are only achievable with reduced image resolution. There is an additional compromise between frame rate and maximal exposure, with higher imaging speeds suffering from reduced image contrast. After preliminary tests, a frame rate of $50,000 \mathrm{fps}$ was settled on for collecting schlieren footage, as this allowed a good spatial resolution and the image was not limited by a lack of light when a maximal exposure time was used $(20 \mu \mathrm{s})$. It should be noted that there is not a direct relationship between the frame rate of the footage and the sampling rate of the recovered audio, and 50,000 fps does not limit audio recovery to a Nyquist frequency of $25 \mathrm{kHz}$. Rather, the effective sampling rate will be dependent on the spatial sampling rate of the image, itself a function of the image magnification and wave speed, as well as the smoothing and downsampling carried out in the processing.

To experimentally evaluate the impact of imaging parameters on signal recoverability, exposure time, frame rate, and spatial resolution were varied. Variations in exposure (4-20 $\mu \mathrm{s})$ and frame rate (40,000-60,000 fps) were found to have little effect on recovering a $10 \mathrm{kHz}$ wave; with SNRs remaining between 8 and 12. Footage was also collected with a different setup that prioritized spatial resolution over temporal resolution (frame rate), with an image of $424 \mathrm{x}$ 512 pixels recorded at $31,000 \mathrm{fps}$, instead of the standard resolution of $256 \times 480$ pixels recorded at 50,000 fps. While signals were still recoverable, this resulted in a dramatic worsening of SNR to -10 and below. This suggests that the system could be further optimized by changing the imaging parameters in the other direction, increasing temporal resolution at the cost of spatial resolution. 


\section{Discussion}

The origins of sound visualization go back as far as the phonautrograph of douard-Lon Scott de Martinville, patented in 1857. While the phonautograph produced visual outputs for the inspection of acoustic information, it was only after Thomas Edison's invention of the phonograph that the focus of acoustic engineering shifted from visualizing sound to recovering audio information for playback and analysis. With the work presented here, a similar shift is made for a different imaging modality of acoustic phenomena.

For a broad spectrum of acoustic waves, we show that schlieren imaging performed with an inexpensive apparatus is capable of recovering significant amounts of original audio information. Our approach has several benefits and potential applications. As a general exercise in how to gather quantitative data from schlieren images, we present a detailed consideration of the image manipulation, processing, and analysis that retains and recovers the original information within the image. The schlieren microphone is capable of detecting a sound field while external to it, and therefore does not disrupt the propagation of acoustic waves. This may have particular relevance to acoustic refraction or other studies of acoustic wave propagation, as well as in assessing the qualities of acoustic output for audio devices such as speakers. The work described here was performed an inexpensive mirror, and without the use of optical benches. With higher quality optical components and a more tunable apparatus, a schlieren microphone of greater sensitivity would have many more potential applications. Even with components of higher specification, a high-speed schlieren imaging system may be more affordable than coherent light-based techniques such as LDA and LDV, as well as presenting fewer safety risks.

As a general caveat to this work, we wish to state clearly that this is not a microphone in the traditional sense, and would be unsuitable for recording the audio signals most often received by conventional transducer microphones. While the SPLs of sound waves recovered in this work are significantly below the expected threshold of schlieren imaging 'visibility', they are still above the typical conversational speaking volume of $60 \mathrm{~dB}$. Rather, the schlieren microphone will have particular applications where a non-contact method of sound field reconstruction is required.

More abstractly, the schlieren microphone provides another visual modality for the consideration of sound waves, as previously established by techniques such as LDV. This may prove especially valuable for pedagogical considerations of acoustics, optics, and digital signal processing. Both sound and vision are phenomena for which we have natural, though different, intuitions. Sound particularly lends itself to considerations of harmonics and Fourier analysis, as mathematical principles such as filters and superposition can be related to aural experiences of music (resonant acoustic spaces and combining individual notes, respectively). Visual experience and imagination is fundamental for many people's understanding of the world, and particularly useful in comparing numerical data. By uniting both perceptual modalities, the schlieren microphone may serve as a useful conceptual tool for illustrating mathematical and physical principles.

\section{Conclusion}

We have found that schlieren imaging is a promising imaging modality for the study of acoustic phenomena occupying the domain of human hearing. Previous work has largely been confined to discussions of visibility, which is subject to the limitations of the human visual system, particularly for lower-frequency waveforms. By focusing on a computational and image-processing approach, we have extended the scope of acoustic phenomena for which schlieren imaging may have potential applications.

\section{Funding}

JSH is funded by the Andrew W. Mellon Foundation and Clarendon Foundation through the Oxford interdisciplinary research center, TORCH. The equipment used was developed with funding from the Oxford University John Fell Fund. 


\section{Acknowledgments}

The authors wish to thank the researchers of the interdisciplinary Ordered Universe Project (supported by Arts and Humanities Research Council grant AH/N001222/1) for many stimulating discussions on sound, prompted by studying the writings of thirteenth century scientist and bishop, Robert Grosseteste (c. 1170-1253). The initial inspiration for this work came from studying Grosseteste's pioneering text on sound and phonetics, De generatione sonorum [On the Generation of Sound]. We also thank Rebekah White and Sarah Regan for comments on the manuscript.

\section{A. Appendix}

It is mentioned in section 2.1 that schlieren imaging probes the variations in refractive index along the optical path of the system. The technique is therefore a line-integration of these variations through the imaging field. For spherically-propagating sound waves emitted by a speaker acting as an effective point source this may initially raise concerns, as the refractive index variations will be highly heterogeneous throughout the sound field along the optical path. Indeed, this is a significant issue for laser Doppler methods that probe absolute refractive index (the scalar field n), which confounds the task of audio recovery and sound field reconstruction. A discussion of this may be found in [21].

Here, schlieren imaging has an inherent advantage. By probing the first derivative of refractive index (the vector field $\nabla \mathrm{n}$ ), and only in the direction perpendicular to the razor in axes orthogonal to the optical path, the sensitivity of a schlieren system to these heterogeneous refractive inhomogeneities is reduced. We may consider a local change in refractive index encountered by the optical path through the schlieren system, $\nabla \mathrm{n}$, as a three-dimensional vector. The schlieren contribution, $s$, is then the scalar product of this vector and the property of the filter, $f$ :

$$
\boldsymbol{s}=\boldsymbol{f}_{\boldsymbol{y}} \cdot \boldsymbol{\nabla n}=\left[\begin{array}{lll}
0 & 1 & 0
\end{array}\right]\left[\begin{array}{l}
\delta \mathrm{n} / \delta x \\
\delta \mathrm{n} / \delta y \\
\delta \mathrm{n} / \delta z
\end{array}\right] \quad \text { for a } y \text {-perpendicular filter. }
$$

The sensitivity of a schlieren system to $\delta \mathrm{n}$ is therefore scaled according to the decomposition of its vector with respect to the axis perpendicular to the razor, giving $\delta \mathrm{n}(\cos \theta)$ where $\theta$ is the angle to the axis. For a double-pass schlieren system such as is used in this research, this scaling occurs twice, giving $\delta \mathrm{n}(\cos \theta)^{2}$.

This $(\cos \theta)^{2}$ scaling function as modeled for spherical sound waves emitted from a point source is shown in Figure 16 , with a cross-section of the $y z$ plane at $x=0$.

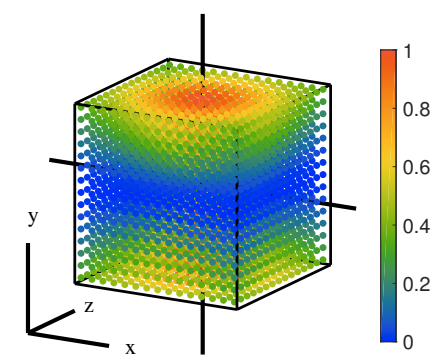

(a)

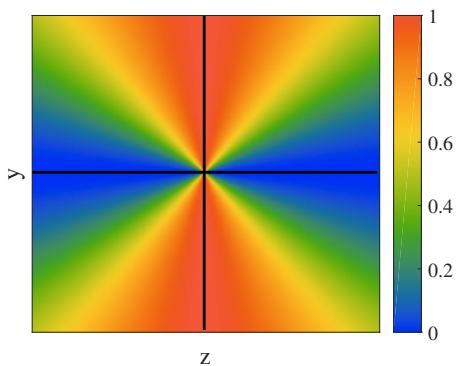

(b)

Figure 16: a) The $(\cos \theta)^{2}$ scaling function for gradients of refractive index along the optical path of the schlieren system. b) A cross-section of the $(\cos \theta)^{2}$ scaling function through the $y z$ plane at $x=0$. 


\section{References}

[1] P. Freymuth, Flow visualization in fluid mechanics, Review of Scientific Instruments 64 (1993) 1-18.

[2] O. C. Bixler, Color Schlieren as a Quantitative Tool for Acoustic Measurements in Ballistics, The Journal of the Acoustical Society of America 44 (1968) 375-375.

[3] M. M. Biss, G. S. Settles, M. J. Hargather, L. J. Dodson, J. D. Miller, High-speed digital shadowgraphy of shock waves from explosions and gunshots, in: Shock Waves, Springer, Berlin, Heidelberg, 2009, pp. 91-96.

[4] D. Mller, N. Degen, J. Dual, Schlieren visualization of ultrasonic standing waves in mm-sized chambers for ultrasonic particle manipulation, Journal of Nanobiotechnology 11 (2013) 21

[5] J. W. Tang, A. D. G. Nicolle, J. Pantelic, M. Jiang, C. Sekhr, D. K. W. Cheong, K. W. Tham, Qualitative Real-Time Schlieren and Shadowgraph Imaging of Human Exhaled Airflows: An Aid to Aerosol Infection Control, PLOS ONE 6 (2011) e21392.

[6] J. Rowell, M. Noguchi, B. M. Bernhardt, A. Herdman, B. Gick, M. Schellenberg, Schlieren study of external airflow during the production of nasal and oral vowels in French, Canadian Acoustics 44 (2016).

[7] J. A. Westphal, Schlieren Technique for Studying Water Flow in Marine Animals, Science 149 (1965) $1515-1516$.

[8] G. S. Settles, Schlieren and Shadowgraph Techniques: Visualizing Phenomena in Transparent Media, Springer Science \& Business Media, 2001.

[9] G. S. Settles, M. J. Hargather, A review of recent developments in schlieren and shadowgraph techniques, Measurement Science and Technology 28 (2017) 042001.

[10] M. J. Hargather, G. S. Settles, M. J. Madalis, Schlieren imaging of loud sounds and weak shock waves in air near the limit of visibility, Shock Waves 20 (2010) 9-17.

[11] L. M. Weinstein, K. Stacy, G. J. Vieira, E. A. Haering Jr, A. H. Bowers, Visualization and image processing of aircraft shock wave structures, in: 1st Pacific Symposium on Flow Visualization and Image Processing, Feb. 23-26, 1997, Honolulu, Hawaii.

[12] N. Chitanont, K. Yaginuma, K. Yatabe, Y. Oikawa, Visualization of sound field by means of Schlieren method with spatio-temporal filtering, in: 2015 IEEE International Conference on Acoustics, Speech and Signal Processing (ICASSP), pp. 509-513.

[13] N. Chitanont, K. Yatabe, Y. Oikawa, Audible sound field visualization by using Schlieren technique, in: 12th West. Pac. Acoust. Conf.(WESPAC), pp. 5-9.

[14] N. Chitanont, K. Yatabe, K. Ishikawa, Y. Oikawa, Spatio-temporal filter bank for visualizing audible sound field by Schlieren method, Applied Acoustics 115 (2017) 109-120.

[15] B. Piper, T. Koukoulas, I. Butterworth, Illuminating sound: a review of how sound can be measured using light, in: Institute of Acoustics 40th Anniversary Conference, volume 36.

[16] J. S. Cullen, C. A. Greated, D. M. Campbell, LDA measurement of sound: amplitude modulation of laser Doppler signals, Measurement Science and Technology 10 (1999) 812.

[17] T. Macgillivray, D. Campbell, C. Greated, R. Barham, The Development of a Microphone Calibration Technique Using Laser Doppler Anemometry, Acta Acustica united with Acustica 88 (2002) 135-141.

[18] M. W. Thompson, A. A. Atchley, Simultaneous measurement of acoustic and streaming velocities in a standing wave using laser Doppler anemometry, The Journal of the Acoustical Society of America 117 (2005) 1828-1838.

[19] T. Koukoulas, P. Theobald, T. Schlicke, R. Barham, Towards a future primary method for microphone calibration: Optical measurement of acoustic velocity in low seeding conditions, Optics and Lasers in Engineering 46 (2008) 791-796.

[20] B. Gazengel, S. Poggi, Measurement of acoustic particle velocities in enclosed sound field: Assessment of two Laser Doppler Velocimetry measuring systems, Applied Acoustics 66 (2005) 15-44

[21] R. Malkin, T. Todd, D. Robert, Quantitative imaging of acoustic reflection and interference, Journal of Physics: Conference Series 581 (2015) 012007.

[22] J. P. Sharpe, C. A. Greated, The measurement of periodic acoustic fields using photon correlation spectroscopy, Journal of Physics D: Applied Physics 20 (1987) 418.

[23] J. P. Sharpe, C. A. Greated, A stochastic model for photon correlation measurements in sound fields, Journal of Physics D: Applied Physics 22 (1989) 1429.

[24] A. Davis, M. Rubinstein, N. Wadhwa, G. Mysore, F. Durand, W. T. Freeman, The Visual Microphone: Passive Recovery of Sound from Video, ACM Transactions on Graphics (Proc. SIGGRAPH) 33 (2014) 79:1-79:10.

[25] H. G. Taylor, J. M. Waldram, Improvements in the Schlieren method, Journal of Scientific Instruments 10 (1933) 378.

[26] J. S. Harvey, H. E. Smithson, C. R. Siviour, Data for: Visualization of acoustic waves in air and subsequent audio recovery with a high-speed schlieren imaging system (Harvey et al.) 1 (2017). Https://data.mendeley.com/, DOI: http://dx.doi.org/10.17632/mjrx5467mv.1.

[27] K. K. Paliwal, L. Alsteris, Usefulness of phase spectrum in human speech perception, in: Eighth European Conference on Speech Communication and Technology.

[28] Guangji Shi, M. Shanechi, P. Aarabi, On the importance of phase in human speech recognition, IEEE Transactions on Audio, Speech and Language Processing 14 (2006) 1867-1874.

[29] F. W. Campbell, J. G. Robson, Application of fourier analysis to the visibility of gratings, The Journal of Physiology 197 (1968) $551-566$.

[30] J. Mannos, D. Sakrison, The effects of a visual fidelity criterion of the encoding of images, IEEE Transactions on Information Theory 20 (1974) 525-536. 